\title{
HIGH INCIDENCE OF MULTIRESISTANT BACTERIAL ISOLATES FROM BLOODSTREAM INFECTIONS IN TRAUMA EMERGENCY DEPARTMENT AND INTENSIVE CARE UNIT IN SERBIA
}

\author{
OLIVERA DJURIC ${ }^{1,2 *}$, LJILJANA MARKOVIC-DENIC ${ }^{1,2}$, BOJAN JOVANOVIC ${ }^{1,3}$ and \\ VESNA BUMBASIREVIC ${ }^{1,3}$ \\ ${ }^{1}$ Faculty of Medicine, University of Belgrade, Belgrade, Serbia \\ ${ }^{2}$ Institute of Epidemiology, Faculty of Medicine, University of Belgrade, Belgrade, Serbia \\ ${ }^{3}$ Center for Anaesthesiology, Emergency Center, Clinical Center of Serbia, \\ Belgrade, Serbia
}

(Received: 23 November 2018; accepted: 18 December 2018)

We investigated the incidence of bloodstream infections (BSIs) in trauma emergency department (ED) and intensive care unit (ICU), to assess ED- and ICUrelated predictors of BSI and to describe the most common bacteria causing BSI and their antimicrobial resistance markers. A prospective study was conducted in two trauma ICUs of the ED of Clinical Center of Serbia. Overall, 62 BSIs were diagnosed in 406 patients, of which 13 were catheter-related BSI (3.0/1,000 CVC-days) and 30 BSIs of unknown origin, while $15 \%$ were attributed to ED CVC exposure. Lactate $\geq 2 \mathrm{mmol} / \mathrm{L}$ and SOFA score were independent ED-related predictors of BSI, while CVC in place for $>7$ days and mechanical ventilation $>7$ days were significant ICUrelated predictors. The most common bacteria recovered were Acinetobacter spp., Klebsiella spp., and Pseudomonas aeruginosa. All Staphylococcus aureus and coagulase-negative staphylococci isolates were methicillin-resistant, whereas $66 \%$ of Enterococcus spp. were vancomycin-resistant. All isolates of Enterobacteriaceae were resistant to third-generation cephalosporins, whereas $87.5 \%$ of $P$. aeruginosa and 95.8\% of Acinetobacter spp. isolates were resistant to carbapenems. ED BSI contributes substantially to overall ICU incidence of BSI. Lactate level and SOFA score can help to identify patients with higher risk of developing BSI. Better overall and CVC-specific control measures in patients with trauma are needed.

Keywords: trauma, bloodstream infections, antimicrobial resistance, emergency department, intensive care unit

*Corresponding author; E-mail: oliveradjuric87@gmail.com 


\section{Introduction}

Healthcare-associated bloodstream infections (HA-BSIs) are considered as major preventable threat to healthcare and patient safety. They approximately affect $4 \%$ of all critically ill patients and are the second most common healthcareassociated infection (HAI) among patients staying more than $48 \mathrm{~h}$ in intensive care unit (ICU) [1]. Moreover, HA-BSI is independently associated with increased risk of both short- and long-term mortality in critically ill patients [2, 3].

Patients with trauma represent considerable part of patients admitted in emergency department (ED) since the advances in initial resuscitation of critically injured patients increased survival after the injury. However, those who survive initial trauma are at particular risk of developing BSI over the course of ED and ICU treatment, which is enabled by exposure to numerous invasive devices and facilitated by disrupted barriers and altered immune response [4]. Consequently, BSI is two times more frequent in patients with trauma compared to surgical patients and after ventilator-associated pneumonia, it is the second most frequent HAI in patients with trauma [5, 6]. Yet, surveillance studies and preventative measures usually target BSI in ICU inpatients and little is known about contribution of ED in occurrence of BSI. In Serbia, trauma represents the third cause of mortality and every tenth person dies from injury [7]. Despite this, there is neither in depth data on incidence of BSI in patients with trauma in both ED or ICU department in Serbia nor the countries of the Southeastern-European Region.

In critically ill patients, healthcare-acquired BSI is usually accompanied with sepsis, which has significant impact on patient's clinical course, since primary bacteremia is associated with higher mortality compared with pulmonary and abdominal sources of sepsis [8]. Clinicians mostly rely on positive blood culture in early diagnosis of BSI and sepsis as it helps distinguishing infectious from non-infectious inflammatory response and allows optimization of empirical antimicrobial therapy. However, even when requested on time, blood culture requires up to $48 \mathrm{~h}$ to yield results, while every hour of the delay of the antibiotics initiation in patients with sepsis increases risk for unfavorable outcome [9]. Hence, search for factors available in first hours of admission to ED could help to select patient at risk for development of BSI and sepsis later over the course of ED and ICU treatment. Moreover, choosing the adequate empirical antimicrobial therapy requires knowledge about predominant causative agents and their resistance patterns in particular group of patients and medical setting. This is allowed by constant update of emergency care providers on main antimicrobial markers of pathogens causing BSI, as it has been shown that feedback of surveillance data to clinical staff is significant tool in reducing the rates of HAI [10]. 
Therefore, the aims of this study were: (a) to estimate incidence of bacterial BSI in ICU patients with trauma and fraction of BSI cases attributed to ED; (b) to identify which factors among those assessed in the first hours in ED and those estimated later on in ICU are independent predictors of BSI in patients with trauma; and (c) to estimate most common bacteria causing BSI and their most common resistance markers in patients with trauma in Emergency Center in Serbia. To assess this, we used patients with trauma as a pilot population since they are at highest risk of acquiring HAI, and moreover, those who sustained trauma are considered not to have infection on the admission.

\section{Materials and Methods}

\section{Study setting and infection control measures}

Emergency Department of Clinical Center of Serbia is a 308-bed tertiary teaching hospital and the sole referral and adult care trauma center serving the southern and central areas of Serbia. Two trauma-surgical ICUs together comprise 25 beds and have approximately 800 admissions annually. Nurse-to-patient ratio is 1:3. Hand hygiene policy includes alcohol-based hand sanitizers placed at each bedside in each ICU room, according to the national recommendations, which are adopted from World Health Organization's hand hygiene guidelines [11]. Central venous catheter (CVC) insertion and maintenance are done following the guidelines of American Society of Anesthesiologists for insertion and maintenance of CVC [12]. Placement of the CVC is done in aseptic conditions either by the anesthesiology specialist or by anesthesiology resident under the supervision of a specialist. Hand hygiene (using alcohol-based hand rubs), universal barrier precautions (use of a cap, mask, sterile gown, sterile gloves, and a sterile partial-body drape), and skin preparation (using chlorhexidine) are performed prior and during every CVC insertion.

The infection control team in the ED was established in 2001 within the Department for HAI at the Clinical Center of Serbia. It comprises one infection control epidemiologist and one infection control nurse who deliver general and specific infection control measures recommended by the National Infection Control Committee. No modifications in infection control measures were observed during the study period.

\section{Study design and patients}

A prospective surveillance study was conducted at the Emergency Department of Clinical Center of Serbia in a year and a half period (from November 2014 
to April 2016). All consecutive adult patients with trauma were admitted to one of two trauma ICUs from ED and those who spent more than $48 \mathrm{~h}$ in ICU were eligible for the study. Patients were transferred either from the ED directly to the ICU or via operating room if urgent surgery was indicated. Exclusion criteria were unspecified injuries, non-traumatic injures (poisoning, drowning, and suffocation), hip fractures, late effect of injury, superficial injuries, and foreign bodies. Patients with isolated brain injuries caused by low falls due to non-traumatic intracranial hemorrhage were also excluded.

The study was approved by the Ethics Committee of the Clinical Center of Serbia (no. 1358/19) and by the Ethics Committee of the Faculty of Medicine at the University of Belgrade, Serbia (no. 29/X-5).

\section{Surveillance and case definitions}

Patients were prospectively assessed for the presence of BSI by daily visits by attending physicians and infection control epidemiologists and examination of records of patients, clinical course, and microbiological results. The centers for disease and prevention definitions were used to diagnose BSI [13]. BSI was diagnosed when clinical symptoms of infection were present $48 \mathrm{~h}$ from admission, and laboratory-confirmed causative agent was isolated, i.e., recognized pathogen cultured from one or more blood cultures or common skin contaminant cultured from two or more blood cultures.

BSIs were reported as primary and secondary BSI. Primary BSI was defined as BSI not related to another infection site and for the purpose of the comparability of results with studies, which have used European Centre for Disease Prevention and Control criteria, these were divided into catheter-related BSI (CR-BSI) and BSI of unknown origin $[14,15]$. All other cases of BSI, i.e., those related to another infection site (same organism cultured from bloodstream and primary HAI site, and the organisms exhibit same antibiogram) were considered as secondary BSI [16]. A microbiological proof of a catheter-related infection was warranted when the same organism was isolated from a peripheral blood culture and a catheter blood or as a positive semi-quantitative CVC culture ( $>15 \mathrm{CFU}$ per catheter segment).

The extent of exposure to CVC was described as device utilization ratio, calculated by dividing the total number of CVC-days by total number of patientdays. The presence of multiple CVCs in a single patient on a single day was represented as one CVC-day. Cumulative incidence (number of BSI episodes per 100 patients) and incidence rate (number of BSI per 1,000 patient-days or 1,000 CVC-days) were calculated as outcome measures. 


\section{Definitions}

Severity of trauma was assessed through the Abbreviated Injury Scale (AIS) and Injury Severity Score (ISS). Glasgow Coma Scale (GCS) was used to measure conscious level before sedation. Severe trauma brain injury (TBI) was defined as AIShead $\geq 3$ and GCS $\leq 8$. The Acute Physiology and Chronic Health Evaluation II score (APACHE II) and Sequential Organ Failure Assessment (SOFA) score are a severity-of-disease classification scores and were calculated within $24 \mathrm{~h}$ of admission of a patient in ED. Comorbidity polypharmacy score was defined as the sum of total number of pre-admission medications and the total number of comorbidities for each patient [17].

\section{Microbiological assessment}

Isolation and identification of bacterial strains were carried out following standard microbiological procedures. Antimicrobial susceptibility was performed using the Kirby-Bauer disk diffusion method and a Vitek2 automated system (bioMérieux, Marcy-I'Etoile, France). Zone diameter was measured and interpreted according to the Clinical and Laboratory Standards Institute guidelines [18]. Strains that showed intermediate susceptibility and resistance to the specific antibiotic were considered resistant. Antimicrobial resistance (AMR) was presented through the AMR markers as follows: for Staphylococcus aureus: markers of methicillin (oxacillin, cefoxitin, or methicillin) resistance - methicillin-resistant Staphylococcus aureus; for Enterococcus spp.: markers of glycopeptide resistance vancomycin-resistant enterococci; for Enterobacteriaceae: third-generation cephalosporins (ceftriaxone, cefotaxime, or ceftazidime) and carbapenems (imipenem, meropenem or doripenem, and ertapenem) resistance; and for Gram-negative rods (Pseudomonas aeruginosa and Acinetobacter spp.): antipseudomonal carbapenems (imipenem or meropenem) and colistin resistance.

\section{Statistical analysis}

For continuous variables, the Kolmogorov-Smirnov test was used to assess the assumption of normality. Continuous data are presented as the mean \pm standard deviation or median (interquartile range) and categorical data are presented as numbers (percentages). Univariate analysis of significant difference between groups of patients with and without BSI for continuous data was performed using Student's $t$ test for normally distributed data or Mann-Whitney $U$ test for skewed data, and for categorical data using the Pearson's $\chi^{2}$ test 
(less than $20 \%$ of cells with expected frequencies $<5$ and no cell has expected frequency $<1$ ) or Fisher's exact test (for $2 \times 2$ contingency tables where more than $20 \%$ of cells have expected frequencies $<5$ ). A multivariate logistic regression was applied to indentify independent predictors of BSI. Variables were entered into the multivariate logistic regression model based on statistical significance $(p<0.25$ in univariate analysis) and clinical significance, and stepwise logistic regression was performed. Odds ratios (ORs) with 95\% confidence intervals (95\% CIs) were computed. Hosmer-Lemeshow goodness-of-fit test and area under the receiveroperating characteristic curve (AUC) were applied to assess overall model fit. C statistics was calculated for two distinct models (with and without predictors from ED) as a measure of discrimination of BSI and non-BSI cases and comparison of AUCs was done using DeLong's method [19]. All statistical tests were two-sided and were performed at a 5\% significance level. Statistical analyses were performed using SPSS version 20.0 software (IBM-SPSS Inc., Armonk, NY, USA).

\section{Results}

\section{Study population}

During the study period, 848 patients with trauma were admitted to ED and consequently to one of two trauma-surgical ICUs, of which 547 were adult patients who were hospitalized for more than $48 \mathrm{~h}$. Thirty-one patients were excluded due to non-traumatic mechanisms of injury (poisoning, drowning, and suffocation) and 36 were excluded due to late effect of injuries, superficial injuries, or foreign bodies. In addition, 74 patients were excluded due to diagnosis of burns, hip fractures, or isolated brain injuries caused by non-traumatic injury, such as spontaneous intracranial bleeding. This led to the sample of 406 patients with trauma included in the final analysis. The process of patient selection is depicted in Figure 1.

A cohort of 406 patients with trauma (312 males and 94 females) were followed for 5,258 patient-ICU days. Median length of stay was 9 days (range: 2-131).

\section{Incidence of BSI in ED and ICU}

Out of 406 patients with trauma, 57 patients had at least one episode of BSI (cumulative incidence 14.0\%). Overall, 62 BSI episodes were diagnosed in 57 patients with the incidence rate of 11.8/1,000 patient/days. Out of 62 BSI episodes, $19(30.6 \%)$ BSI episodes infection cite was identified and those were considered as secondary BSI. For 30 BSI episodes (48.4\%), no source of infection could be 


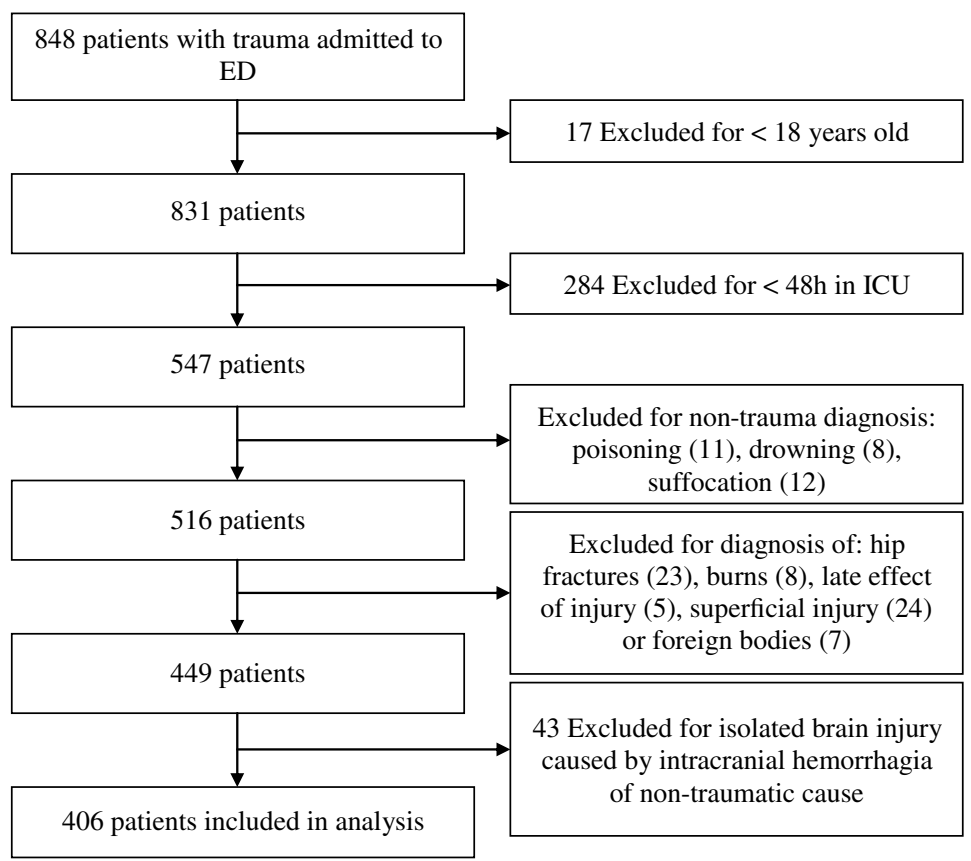

Figure 1. Flowchart of patient selection

identified while 13 BSI (21.0\%) were ascertained as CR-BSI, which over the 4,295 catheter-days resulted in incidence rate of 3.0/1,000 CVC-days. Out of 13 CR-BSI, 2 were attributed to ED since the $\mathrm{CVC}$ was placed in $\mathrm{ED}$, which resulted in $\mathrm{IR}=3.7 / 1,000 \mathrm{CVC} /$ days $(532 \mathrm{CVC}$-days in $\mathrm{ED})$.

\section{Clinical characteristics of patients at admission to ED}

Table I provides comparison of demographic characteristics, underlying conditions, and data about severity of the disease at the admission to ED, between patient with and without BSI. There was no significant difference in demographics and comorbidities in patients with BSI and those without BSI. However, significantly more patients with BSI had received cardiopulmonary resuscitation on scene or at admission $(p=0.026)$, had lower median GCS on admission $(p=0.001)$, had almost twice more patients with lactate level $\geq 2 \mathrm{mmol} / \mathrm{L}$ $(p<0.001)$ and in higher percent required mechanical ventilation $(p<0.001)$ as well as massive transfusion $(p=0.011)$ and emergency surgery $(p=0.034)$ on admission. Patients with BSI evinced more severe medical condition on admission 
Table I. Characteristics of patients with trauma with and without BSI

\begin{tabular}{|c|c|c|c|c|}
\hline Characteristic & $\begin{array}{l}\text { All patients } \\
\quad(n=406)\end{array}$ & $\begin{array}{c}\text { BSI } \\
(n=57)\end{array}$ & $\begin{array}{l}\text { Non-BSI } \\
(n=349)\end{array}$ & $p$ value \\
\hline \multicolumn{5}{|l|}{ General characteristics } \\
\hline Age & $49(35)$ & $52(37)$ & $48(34)$ & 0.525 \\
\hline Male gender & $312(76.8)$ & $44(77.2)$ & $268(76.8)$ & 0.947 \\
\hline Comorbidities & $165(40.6)$ & $23(40.4)$ & $142(40.7)$ & 1.000 \\
\hline Cardiac disease & $94(23.2)$ & $17(29.8)$ & $77(22.1)$ & 0.235 \\
\hline Chronic pulmonary disease & $12(3.0)$ & $2(3.5)$ & $10(2.9)$ & 0.679 \\
\hline Chronic liver disease & $3(0.7)$ & $0(0.0)$ & $3(0.9)$ & 1.000 \\
\hline Chronic renal disease & $4(1.0)$ & $1(1.8)$ & $3(0.9)$ & 0.455 \\
\hline Diabetes mellitus & $16(3.9)$ & $3(5.3)$ & $13(3.7)$ & 0.480 \\
\hline Immunocompromised & $6(1.7)$ & $0(0.0)$ & $6(1.7)$ & 1.000 \\
\hline Carcinoma & $10(2.5)$ & $0(0.0)$ & $10(2.9)$ & 0.369 \\
\hline \multicolumn{5}{|l|}{ Number of comorbidities } \\
\hline 0 & $241(59.4)$ & $34(59.6)$ & $207(59.3)$ & 0.883 \\
\hline 1 & $111(27.3)$ & $17(29.8)$ & $94(26.9)$ & \\
\hline 2 & $32(7.9)$ & $4(7.0)$ & $28(8.0)$ & \\
\hline$\geq 3$ & $22(5.4)$ & $2(3.5)$ & $20(5.7)$ & \\
\hline Comorbidity polypharmacy score & $1.6 \pm 2.5$ & $1.8 \pm 2.6$ & $1.6 \pm 3.0$ & 0.519 \\
\hline \multicolumn{5}{|c|}{ Clinical characteristics at admission to ED } \\
\hline \multicolumn{5}{|c|}{ Type of admission } \\
\hline ED directly & $290(71.4)$ & $34(59.6)$ & $256(73.4)$ & 0.034 \\
\hline OR & $116(28.6)$ & $23(40.4)$ & $93(26.6)$ & \\
\hline Glasgow coma score & $14(6)$ & $12(6)$ & $14(5)$ & 0.001 \\
\hline$\leq 8$ & $87(21.5)$ & $15(26.3)$ & $72(20.7)$ & 0.033 \\
\hline $9-12$ & $61(15.1)$ & $914(24.6)$ & $47(13.5)$ & \\
\hline$\geq 13$ & $257(63.5)$ & $28(49.1)$ & $229(65.8)$ & \\
\hline Lactate $(\mathrm{mmol} / \mathrm{L})$ & $1.5(1.8)$ & $2.3(3.1)$ & $1.4(1.7)$ & $<0.001$ \\
\hline Lactate $\geq 2 \mathrm{mmol} / \mathrm{L}$ & $160(39.4)$ & $36(63.2)$ & $124(35.5)$ & $<0.001$ \\
\hline Mechanical ventilation & $236(58.1)$ & 45 (78.9) & $191(54.7)$ & $<0.001$ \\
\hline \multicolumn{5}{|l|}{ Intubation place } \\
\hline At scene & $12(5.1)$ & $0(0.0)$ & $12(6.3)$ & 0.095 \\
\hline $\mathrm{ED} / \mathrm{OR}$ & $160(67.8)$ & $37(82.2)$ & $123(64.4)$ & \\
\hline ICU & $14(5.9)$ & $2(4.4)$ & $12(6.3)$ & \\
\hline Other hospital & $50(21.2)$ & $6(13.3)$ & $44(23.0)$ & \\
\hline \multicolumn{5}{|l|}{ State on admission } \\
\hline Coma & $76(18.7)$ & $14(24.6)$ & $62(17.8)$ & 0.223 \\
\hline Hypotension & $67(16.5)$ & $17(29.8)$ & $50(14.3)$ & 0.003 \\
\hline Hemorrhagic shock & $24(5.9)$ & $9(15.8)$ & $15(4.3)$ & 0.003 \\
\hline Massive transfusion & $15(3.7)$ & $6(10.5)$ & $9(2.6)$ & 0.011 \\
\hline CPR & $9(2.2)$ & $4(7.0)$ & $5(1.4)$ & 0.026 \\
\hline Emergency surgery & $116(28.6)$ & $23(40.4)$ & $93(26.6)$ & 0.034 \\
\hline Abdominal surgery & $56(13.8)$ & $14(24.6)$ & $42(12.0)$ & 0.011 \\
\hline Splenectomy & $27(6.7)$ & $7(12.3)$ & $20(5.7)$ & 0.066 \\
\hline Neurosurgery & $28(6.9)$ & $1(1.8)$ & $27(7.7)$ & 0.154 \\
\hline Thoracic surgery & $3(0.7)$ & $0(0.0)$ & $3(0.9)$ & 1.000 \\
\hline Pelvic surgery & $6(1.5)$ & $2(3.5)$ & $4(1.1)$ & 0.200 \\
\hline Maxillofacial surgery & $1(0.2)$ & $1(1.8)$ & $0(0.0)$ & 0.140 \\
\hline Orthopedic surgery & $23(5.7)$ & $4(7.0)$ & $19(5.4)$ & 0.547 \\
\hline
\end{tabular}


Table I. Characteristics of patients with trauma with and without BSI (Continued)

\begin{tabular}{lcccr}
\hline Characteristic & $\begin{array}{c}\text { All patients } \\
(n=406)\end{array}$ & $\begin{array}{c}\text { BSI } \\
(n=57)\end{array}$ & $\begin{array}{c}\text { Non-BSI } \\
(n=349)\end{array}$ & $p$ value \\
\hline Other surgery & $13(3.2)$ & $3(5.3)$ & $10(2.9)$ & 0.340 \\
APACHE II score & $9(10)$ & $12(9)$ & $8(9)$ & $<0.001$ \\
SOFA score & $4(3)$ & $6(4)$ & $3(3)$ & $<0.001$ \\
Device exposure & $7(9)$ & $16(24)$ & $6(8)$ & $<0.001$ \\
CVC-days - median & $326(80.3)$ & $65(100)$ & $261(76.5)$ & $<0.001$ \\
CVC-days $>$ 2 days & $3(7)$ & $13(14)$ & $2(5)$ & $<0.001$ \\
MV-days - median & $197(48.5)$ & $50(87.7)$ & $147(42.1)$ & $<0.001$ \\
CVC-days $>7$ days & & & \\
\hline
\end{tabular}

Note: Values are number (\%), mean $\pm \mathrm{SD}$ or median (IQR). $p$ value corresponds to comparison between BSI and non-BSI. BSI: bloodstream infection; ED: emergency department; OR: operating room; CPR: cardiopulmonary resuscitation; APACHE II score: Acute Physiology and Chronic Health Evaluation II Score; SOFA score: Sequential Organ Failure Assessment score; CVC: central venous catheter; MV: mechanical ventilation.

considering higher median APACHE II and SOFA score in BSI group $(p<0.001$ for both). Regarding the exposure to invasive devices over the course of ED and ICU treatment, median number of days spent with CVC in place or on mechanical ventilation was significantly higher in patients who acquired BSI compared to those who did not ( $p<0.001$ for both).

Table II shows distribution and comparison of injury patterns and severity in patients with trauma with and without BSI. Blunt trauma was a more common type of injury in both groups of patients. Polytrauma $(p<0.001)$ and abdominal injury $(p<0.001)$ were more frequent injury pattern in patients with BSI. They also had significantly more severe injuries on face $(p=0.017)$, abdomen $(p<0.001)$, and external body surface $(p=0.037)$ according to AIS score $\geq 3$ for these body regions. Overall, patients with BSI had more severe injuries according to higher ISS $(24.1 \pm 8.3$ vs. $19.3 \pm 8.4, p<0.001)$ and significantly higher percent of BSI patient had critically severe injuries $(45.6 \%$ vs. $24.6 \%, p=0.002)$.

\section{Predictors of BSI}

Multivariate logistic regression of factors associated with BSI was applied and model was started with 34 variables that were statistically or clinically associated with BSI in univariate analysis. Variables that remained independently associated with occurrence of BSI in multivariate logistic regression model were lactate concentration $\geq 2 \mathrm{mmol} / \mathrm{L}(\mathrm{OR}=2.05 ; 95 \% \mathrm{CI}=1.07-43.89)$, SOFA score $(\mathrm{OR}=1.21 ; 95 \% \mathrm{CI}=1.07-1.36), \mathrm{CVC}$ in place for more than 7 days $(\mathrm{OR}=4.94$; $95 \% \mathrm{CI}=1.97-12.26)$, and mechanical ventilation more than 7 days $(\mathrm{OR}=2.69$; 
Table II. Characteristics of injuries in patients with trauma with and without BSI

\begin{tabular}{|c|c|c|c|c|}
\hline Characteristic & $\begin{array}{l}\text { All patients } \\
(n=406)\end{array}$ & BSI $(n=65)$ & $\begin{array}{l}\text { Non-BSI } \\
(n=341)\end{array}$ & $p$ value \\
\hline Type of injury & & & & 0.322 \\
\hline Blunt & $387(95.3)$ & $53(93.0)$ & $334(95.7)$ & \\
\hline Penetrating & $19(4.7)$ & $4(7.0)$ & $15(4.3)$ & \\
\hline Mechanism of injury & & & & 0.013 \\
\hline Road traffic accidents & $228(56.2)$ & $41(71.9)$ & $187(53.6)$ & \\
\hline Fall from the height & $74(18.2)$ & $6(10.5)$ & $68(19.5)$ & \\
\hline Low fall & $39(9.6)$ & $1(1.8)$ & $38(10.9)$ & \\
\hline Home and leisure injury & $21(5.2)$ & $2(3.5)$ & $19(5.4)$ & \\
\hline Gun shoot injury & $13(3.2)$ & $4(7.0)$ & $9(2.6)$ & \\
\hline Physical assault & $14(3.4)$ & $3(5.3)$ & $11(3.2)$ & \\
\hline Other & $17(4.2)$ & $0(0.0)$ & $17(4.9)$ & \\
\hline Injury pattern & & & & 0.231 \\
\hline Isolated TBI & $29(7.1)$ & $1(1.8)$ & $28(8.0)$ & \\
\hline $\mathrm{TBI}+$ other injuries & $205(50.5)$ & $31(54.4)$ & $174(49.9)$ & \\
\hline Polytrauma without TBI & $172(42.4)$ & $25(43.9)$ & $147(42.1)$ & \\
\hline No. of injured body regions & & & & $<0.001$ \\
\hline 1 & $43(10.6)$ & $1(1.8)$ & $42(12.0)$ & \\
\hline 2 & $101(24.9)$ & $6(10.5)$ & $95(27.2)$ & \\
\hline$\geq 3$ & $262(64.5)$ & $50(87.7)$ & $212(60.7)$ & \\
\hline \multicolumn{5}{|l|}{ Distribution of injuries } \\
\hline Head and neck & $234(57.6)$ & $32(56.1)$ & $202(57.9)$ & 0.805 \\
\hline Face & $128(32.5)$ & $19(33.3)$ & $109(31.2)$ & 0.752 \\
\hline Thorax & $270(66.5)$ & $41(71.9)$ & $229(65.6)$ & 0.349 \\
\hline Abdomen & $103(25.4)$ & $27(47.4)$ & $76(21.8)$ & $<0.001$ \\
\hline Extremities/pelvis & $212(52.2)$ & $36(63.2)$ & $176(50.4)$ & 0.074 \\
\hline Spine & $137(33.7)$ & $20(35.1)$ & $117(33.5)$ & 0.817 \\
\hline External & $133(32.8)$ & $25(43.9)$ & $18(30.9)$ & 0.054 \\
\hline \multicolumn{5}{|l|}{ Injury severity } \\
\hline $\mathrm{AIS}_{\text {head and neck }} \geq 3$ & $179(44.1)$ & $23(40.4)$ & $156(44.7)$ & 0.540 \\
\hline $\mathrm{AIS}_{\text {face }} \geq 3$ & $41(10.3)$ & $11(19.3)$ & $31(8.9)$ & 0.017 \\
\hline AIS $_{\text {thorax }} \geq 3$ & $120(29.6)$ & $21(36.8)$ & $99(28.4)$ & 0.194 \\
\hline $\mathrm{AIS}_{\mathrm{abdomen}} \geq 3$ & $77(19.0)$ & $23(40.4)$ & $54(15.5)$ & $<0.001$ \\
\hline $\mathrm{AIS}_{\text {extremities/pelvis }} \geq 3$ & $77(19.0)$ & $11(19.3)$ & $66(18.9)$ & 0.945 \\
\hline $\mathrm{AIS}_{\text {spine }} \geq 3$ & $85(20.9)$ & $11(19.3)$ & $74(21.2)$ & 0.743 \\
\hline $\operatorname{AIS}_{\text {external }} \geq 3$ & $4(1.0)$ & $2(3.5)$ & $2(0.6)$ & 0.037 \\
\hline ISS & $19.9 \pm 8.5$ & $24.1 \pm 8.3$ & $19.3 \pm 8.4$ & $<0.001$ \\
\hline Mild $(<9)$ & $25(6.2)$ & $0(0.0)$ & $25(7.2)$ & 0.002 \\
\hline Moderate (9-15) & $94(23.2)$ & $7(12.3)$ & $87(24.9)$ & \\
\hline Severe (16-24) & $175(43.1)$ & $24(42.1)$ & $151(43.3)$ & \\
\hline Critical $(25-75)$ & $112(27.6)$ & $26(45.6)$ & $86(24.6)$ & \\
\hline
\end{tabular}

Note: $p$ value corresponds to comparison between BSI and non-BSI. BSI: bloodstream infection; TBI: trauma brain injury; AIS: Abbreviated Injury Scale; ISS: Injury Severity Score. 
Table III. Multivariate logistic regression of predictors of BSI in patients with trauma

\begin{tabular}{lccc}
\hline Variable & OR $[95 \% \mathrm{CI}]$ & $p$ value & Adjusted $p$ value* \\
\hline Lactate $\geq 2 \mathrm{mmol} / \mathrm{L}$ & $2.05[1.07-3.89]$ & 0.029 & 0.056 \\
SOFA score at admission & $1.21[1.07-1.36]$ & 0.002 & 0.004 \\
ICU CVC-days $>7$ & $4.94[1.97-12.26]$ & 0.001 & 0.002 \\
MV-days $>7$ & $2.69[1.35-5.38]$ & 0.005 & 0.010 \\
HL-GOF & $\chi^{2}=6,248 ; d f=8$ & 0.620 & 0.643 \\
AUC $[95 \% \mathrm{CI}]$ & $0.838[0.800-0.874]$ & & \\
\hline
\end{tabular}

Note: SOFA score: sequential organ failure assessment score; OR: odds ratio; ICU: intensive care unit; CVC: central venous catheter; HL-GOF: Hosmer and Lemeshow goodness of fit; MV: mechanical ventilation; BSI: bloodstream infection; AUC: area under the receiver-operator characteristic curve; CI: confidence interval.

*Adjusted for age, gender, comorbidities, severe trauma brain injury ( IIS $_{\text {head }} \geq 3$ and GCS $\leq 8$ ), trauma severity (ISS), and hemorrhagic shock.

$95 \% \mathrm{CI}=1.35-5.38$; Table III). The model fit the data properly since the HosmerLemeshow test was insignificant $(p=0.620)$. The discrimination of the model was good according to AUC $=0.838$ (95\% CI =0.800-0.874). However, after controlling for patients' characteristics (age, gender, and comorbidities), injury severity (severe TBI and ISS) and presence of hemorrhagic shock on admission, lactate concentration $\geq 2 \mathrm{mmol} / \mathrm{L}$ did not remain statistically significant predictor $(\mathrm{OR}=1.93 ; 95 \% \mathrm{CI}=0.984-3.795)$.

In order to estimate the impact of ED predictors in the model, we compared AUCs of models with only ICU variables [CVC in place for more than 7 days and mechanical ventilation (MV) more than 7 days] with full model with ED variables added (lactate $\geq 2 \mathrm{mmol} / \mathrm{L}$ and SOFA score). Inclusion of variables from ED significantly improved discriminative accuracy of the model since AUC increased from fair (AUC $=0.785 ; 95 \% \mathrm{CI}=0.741-0.824)$ to good accuracy (AUC = $0.839 ; 95 \% \mathrm{CI}=0.800-0.874)$, respectively $(p=0.0004$; Figure 2$)$.

\section{Causative agents and AMR}

Sixty-nine pathogens were obtained from 62 BSI episodes, of which 6 were polymicrobial ( 5 isolates with 2 bacteria and 1 with 3 bacteria). The most common pathogens recovered were Gram-negative bacteria (Acinetobacter spp., Klebsiella pneumoniae, Pseudomonas spp., and Klebsiella spp.) accounting for $71 \%$ of all isolates (Table IV). AMR markers in bacteria causing BSI in patients with trauma are presented in Table V. While all isolates of $S$. aureus and coagulase-negative staphylococci (CoNS) were methicillin-resistant, 66\% of Enterococcus spp. were resistant to vancomycin. Regarding Enterobacteriaceae, all isolates of Klebsiella spp., K. pneumoniae, Proteus spp., Serratia spp., and Providentia spp. were 


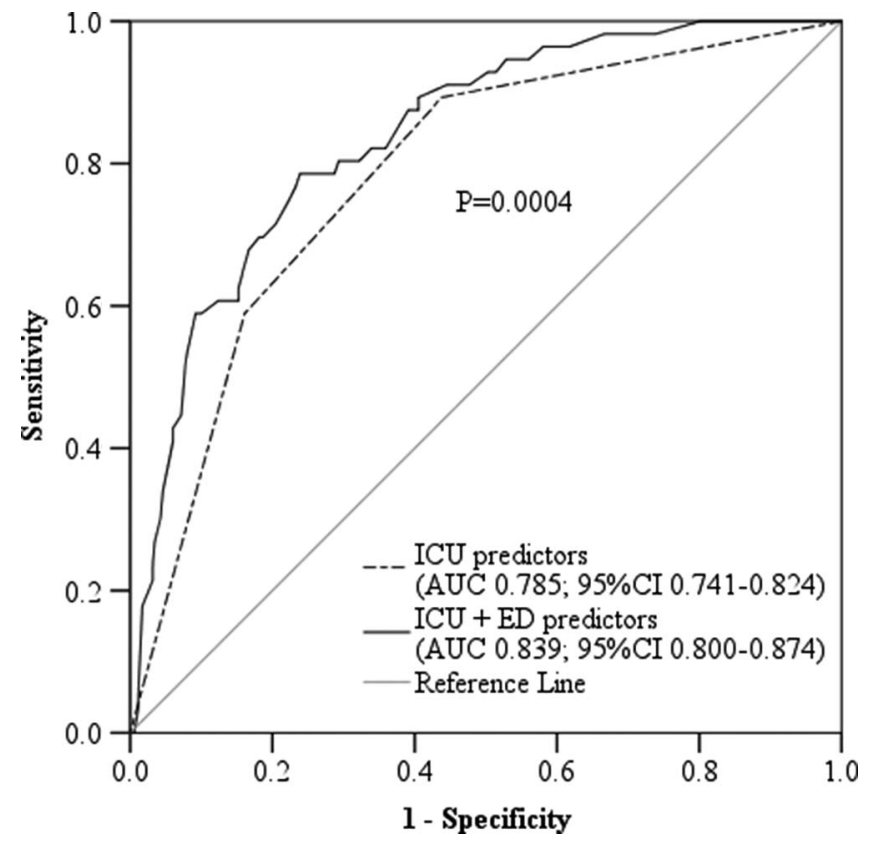

Figure 2. Comparison of AUCs of models with and without ED predictors. AUC difference $=0.055$; $[95 \% \mathrm{CI}=0.0 .3-0.08] ; p=0.0004$

Table IV. Distribution of bacteria causing BSI by type of source

\begin{tabular}{|c|c|c|c|c|}
\hline \multirow[b]{2}{*}{ Pathogen } & \multicolumn{2}{|c|}{ Primary BSI $(n=43)$} & \multirow[b]{2}{*}{$\begin{array}{l}\text { Secondary BSI } \\
\quad(n=19)\end{array}$} & \multirow[b]{2}{*}{ Total } \\
\hline & $\begin{array}{l}\text { CR-BSI } \\
(n=13)\end{array}$ & $\begin{array}{l}\text { Unknown source } \\
\qquad(n=30)\end{array}$ & & \\
\hline S. aureus & $2(15.4)$ & $2(6.1)$ & $2(8.7)$ & $6(8.7)$ \\
\hline Enterococcus spp. & $0(0.0)$ & $5(15.1)$ & $1(4.3)$ & $6(8.7)$ \\
\hline Klebsiella spp. & $1(7.7)$ & $8(24.2)$ & $8(34.7)$ & $17(24.6)$ \\
\hline E. coli & $0(0.0)$ & $0(0.0)$ & $0(0.0)$ & $0(0.0)$ \\
\hline Proteus spp. & $0(0.0)$ & $1(3.0)$ & $0(0.0)$ & $1(1.5)$ \\
\hline Serratia spp. & $0(0.0)$ & $1(3.0)$ & $0(0.0)$ & $1(1.5)$ \\
\hline Providencia spp. & $0(0.0)$ & $2(6.1)$ & $1(4.3)$ & $3(4.3)$ \\
\hline Acinetobacter spp. & $5(38.5)$ & $9(27.3)$ & $10(43.5)$ & $24(34.8)$ \\
\hline Pseudomonas spp. & $4(30.8)$ & $3(9.1)$ & $1(4.3)$ & $8(11.6)$ \\
\hline CoNS & $1(7.7)$ & $2(6.1)$ & $0(0.0)$ & $3(4.3)$ \\
\hline Total & $13(100)$ & $33(100)$ & $23(100)$ & $69(100)$ \\
\hline
\end{tabular}

Note: BSI: bloodstream infection; CR-BSI: catheter-related BSI; CoNS: coagulase-negative staphylococci. 
Table V. AMR markers in bacteria causing BSI in patients with trauma

\begin{tabular}{llcr}
\hline Bacteria & AMR marker & $n$ & $n(\%) R$ \\
\hline S. aureus & MRSA & 6 & $6(100)$ \\
CoNS & MRCoNS & 3 & $3(100)$ \\
Enterococcus spp. & VRE & 6 & $4(66.7)$ \\
Klebsiella spp. & 3GC-NS & 17 & $17(100)$ \\
& CAR-NS & 17 & $13(76.5)$ \\
Proteus spp. & 3GC-NS & 1 & $1(100)$ \\
& CAR-NS & 1 & $0(0.0)$ \\
Serratia spp. & 3GC-NS & 1 & $1(100)$ \\
& CAR-NS & 1 & $0(0.0)$ \\
Providentia spp. & 3GC-NS & 3 & $3(100)$ \\
Pseudomonas spp. & CAR-NS & 3 & $2(66.7)$ \\
Acinetobacter spp. & CAR-NS & 8 & $7(87.5)$ \\
& CST-NS & 8 & $0(0.0)$ \\
& CAR-NS & 24 & $23(95.8)$ \\
Note: AMR: CST-NS & 24 & $4(16.7)$ \\
\hline
\end{tabular}

Note: AMR: antimicrobial resistance; BSI: bloodstream infection; MRSA: methicillin-resistant Staphylococcus aureus; CoNS: coagulase-negative staphylococci; MRCoNS: Methicillin-resistant coagulasenegative staphylococci; VRE: vancomycin-resistant enterococci; 3GC: third-generation cephalosporin; CAR: carbapenem; CST: colistin; NS: non-susceptible.

resistant to third-generation cephalosporins. Considering carbapenem resistance of these bacteria, majority of isolates of Klebsiella spp. (85.7\%), K. pneumoniae (70\%), and Providentia spp. (66.7\%) were denoted as carbapenem resistant, whereas no isolates of Proteus spp. and Seratia spp. were resistant to this class of antimicrobial agents. Gram-negative rods, Pseudomonas spp. and Acinetobacter spp., were highly resistant to carbapenems, as $87.5 \%$ and $95.8 \%$ of their isolates, respectively, were not susceptible to this antibiotic. Of 24 isolates of Acinetobacter spp., 4 (16.7\%) were also resistant to colistin.

\section{Discussion}

In this study, we assessed the incidence of BSI in patients with trauma in ICU of a major referral academic Emergency Center in Belgrade, Serbia, as well as the fraction of BSI, which can be attributed to CVC exposure initiated in ED. In addition, we sought to estimate which of the ED- and ICU-derived variables are independent predictors of BSI and whether ED predictors significantly improve that prediction. At least, we delineated distribution of causative agents of BSI and the major AMR markers of these pathogens. 
Cumulative incidence of BSI in this study was $14 \%$ and the incidence rate was $11.8 / 1,000$ patient/days. Similar to these findings, cumulative incidence in studies that reported BSI as a primary outcome in patients with trauma was $15.2 \%$ in American trauma ICU [20]. However, diverse results were reported within wards and countries where BSI was analyzed as a fraction of all HAIs [21-26].

Regardless of heterogeneity of results, most of the studies focused on incidence and risk factors for BSI acquired in ICU. The reason is that majority of infections present upon admission to ED are community-acquired, whereas those that occur later are usually, due to delayed diagnostic, identified upon admission in ICU. Moreover, according to protocols and guidelines for surveillance studies, if invasive device, such as vascular catheter, is inserted in ED and signs and symptoms of infection occur in ICU, these are reported as ICU-acquired BSI since ED is not considered as inpatient location and for which no denominator data are collected [27]. The consequence is the attribution of ED-acquired HAI to the ICU and underestimation of ED as a significant source of HAI. LeMaster et al. [28] showed that in a study conducted in large urban academic hospital in Boston, based on cases with vascular catheters inserted in ED, incidence of ED BSI is similar to the rates of BSI in ICU. Moreover, Theodoro et al. [29] showed that although ED contributes only $7.7 \%$ of total institutional catheter-days, incidence of central line-associated BSI (CLABSI) in ED is similar to incidence in medical and composite ICUs and it is higher compared to surgical ICU. No such study was conducted in patients with trauma to date, especially not in low or middle income countries. In this study, $15 \%$ of CR-BSIs were acquired in ED with the incidence 3.7/1,000 CVC-days, which is similar to BSI incidence in ICU (3.0/1,000 CVC-days). This can be partially explained by smaller number of CVC-days in ED used as a denominator for incidence calculation. However, the results of this study confirm findings from two previously mentioned studies conducted in unselected population of critically ill patients in terms of considerable fraction of BSI that can be attributed to ED and the similar incidence of BSI acquired in ED and ICU. This is particularly important as it shows that exposure to CVC in ED is significant in developing BSI and necessity of the adaptation of future preventative strategies to include ED in CVC maintenance and hand hygiene guidelines.

We also sought to determine which variables regarding the patient's and trauma characteristic assessed in very first hours of ED admission are associated with later occurrence of BSI together with variables from ICU. Factors identified to independently predict occurrence of BSI in patients with trauma in this study were lactate level $\geq 2 \mathrm{mmol} / \mathrm{L}$ and SOFA score at the admission to ED and CVC in place for more than 7 days and mechanical ventilation for more than 7 days in ICU. This is supported by results of previous studies, which also suggest that severity of disease scores such as APACHE II and SOFA as well as days spent on mechanical 
ventilation and CVC in place is independently associated with HAI and BSI in patients with trauma $[22,25,26]$.

Lactate is widely accepted as marker of hypoperfusion, and its diagnostic value in risk stratification for adverse outcomes has been frequently investigated in diverse subpopulations of critically ill patients [30]. However, the studies mostly focused on unselected ICU patients with suspected or confirmed infection or septic shock and evaluated mortality as an outcome [31]. Several studies addressed this issue within the ED patients and only few with respect to infection prediction. In a study of prediction of bacteremia in adult patients admitted to Swedish ED, Ljungström et al. [32] showed that lactate was significantly higher in patients with bacteremia compared to those without infection $(2.10 \mathrm{vs} .1 .66 \mathrm{mmol} / \mathrm{L})$ and that it has as good predictive ability as a single biomarker as well as in composition with other biomarkers. However, Lin et al. [33] showed that lactate level $19.9 \mathrm{mg} / \mathrm{dl}$ ( $2 \mathrm{mmol} / \mathrm{L}$ ) has slightly better diagnostic accuracy in prediction of Gram-positive bacteremia than procalcitonin (AUC 0.66 vs. 0.61) in adult ED patients, but lower accuracy in predicting Gram-negative bacteremia (AUC 0.71 vs. 0.79 ) and overall positive blood culture (AUC 0.69 vs. 0.72). In this study, however, we do not have in-depth information on other inflammation markers as lactate was selected as marker of tissue damage following trauma and not as an infection diagnostic marker.

In this study, elevated lactate did not remain significant after adjustment for patients' characteristics, trauma severity, hemorrhagic shock, and severe TBI. This result may suggest that increased lactate is only reflecting the severity of the underlying condition motivating the use of $\mathrm{CVC}$, which maintained the strongest association with BSI in adjusted model. Although, in Serbia, CVCs are inserted following CVC guidelines, and CVC management includes change of femoral CVC after 2 days and subclavian and jugular CVC after 7 days of insertion [12], high BSI incidence in ED and ICU can be attributed to possible low compliance to these policies and lack of hygiene measures. Although such measures represent a challenge in life-saving medical setting, they should not be neglected, considering unfavorable outcomes of infections related to CVC use and the fact that great part of CLABSI can be prevented just by adequate adherence to the components of the CL bundles [34].

Considering AMR markers in causative pathogens of BSI, we found alarmingly high rates of Enterobacteriaceae strains resistant to third-generation cephalosporins (100\% of strains) and carbapenems (66.7\%-85.7\%) as well as high percentage of Pseudomonas spp. and Acinetobacter spp. resistant to carbapenems ( $87.5 \%$ and $95.8 \%$ of non-susceptible strains, respectively). On the contrary, National Healthcare Safety Network and International Nosocomial Infection Control Consortium reported considerably lower rates of isolates resistant to common AMR markers $[35,36]$. Although there is no data available on resistance 
to common AMR markers of pathogens causing BSI in patients with trauma from countries close to Serbia, our results are similar to AMR data of invasive isolates in acute care hospitals in countries of Southeastern-European Region [37]. At clinical level, this data is highly important since the choice of empirical therapy of BSI/CLABSI and its duration depend on understanding the distribution and resistance patterns of their causative agents in specific setting or patient population. At national level, this imposes the necessity for implementation of measures for antibiotic consumption restriction together with general and pathogen-specific measures for disruption of transmission of resistant clones.

Several limitations of the study should be addressed. Collecting data from one academic trauma center can be subject to selection bias due to inclusion of mostly severe trauma cases. We did not have data on previous antibiotic consumption due to the urgent type of referral. However, this is the first study in Southeastern-European Region to outline epidemiology of BSI and to estimate contribution of both ED and ICU incidence of BSI as well as contribution of ED variables as risk factors of BSI in population of patients with trauma. Strength of the study is prospective daily collection of numerous clinical and disease severity variables, as well as assessment of signs of infections and the exposure to risk factors with low possibility of misclassification of both exposure and outcome.

In conclusion, BSI is a common HAI in trauma ICU affecting $14 \%$ of all inpatients in ICU. Considerable part of BSI in ICU arises in ED, resulting in similar incidence of BSI in ED compared to ICU. In this study, combination of predictors for BSI assessed in ED and those measured in ICU indicates that constant monitoring of patients on MV and those with CVC for reassessing the need for their continuation may be useful in order to detect primary BSI and pneumonia-related secondary BSI, especially in patients who had hypoperfusion and more severe condition on admission, as estimated by lactate level and SOFA score. Predominance of primary BSI is caused by Gram-negative bacteria highly resistant to commonly used antibiotics, stress necessity for more effective overall, and CVC-specific infection control measures in critically ill patients with trauma.

\section{Acknowledgements}

This work was supported by the Ministry of Education, Science and Technological Development of Serbia, contract no. 175046, 2011-2018.

\section{Conflict of Interest}

The authors declare no conflict of interest related to this work. 


\section{References}

1. European Centre for Disease Prevention and Control: Annual Epidemiological Report 2016 - Healthcare-Associated Infections Acquired In Intensive Care Units [Internet]. ECDC, Stockholm, 2016. Available at https://ecdc.europa.eu/sites/portal/files/documents/ AER-HCAI_ICU_3_0.pdf

2. Wong, S. W., Gantner, D., McGloughlin, S., Leong, T., Worth, L. J., Klintworth, G., Scheinkestel, C., Pilcher, D., Cheng, A. C., Udy, A.: The influence of intensive care unitacquired central line-associated bloodstream infection on in-hospital mortality: A singlecenter risk-adjusted analysis. Am J Infect Control 44, 587-592 (2016).

3. Czaja, A. S., Rivara, F. P., Wang, J., Koepsell, T., Nathens, A. B., Jurkovich, G. J., Mackenzie, E.: Late outcomes of trauma patients with infections during index hospitalization. J Trauma 67, 805-814 (2009).

4. Claridge, J. A., Crabtree, T. D., Pelletier, S. J., Butler, K., Sawyer, R. G., Young, J. S.: Persistent occult hypoperfusion is associated with a significant increase in infection rate and mortality in major trauma patients. J Trauma 48, 8-14 (2000).

5. Wallace, W. C., Cinat, M., Gornick, W. B., Lekawa, M. E., Wilson, S. E.: Nosocomial infections in the surgical intensive care unit: A difference between trauma and surgical patients. Am Surg 65, 987-990 (1999).

6. Glance, L. G., Stone, P. W., Mukamel, D. B., Dick, A. W.: Increases in mortality, length of stay, and cost associated with hospital-acquired infections in trauma patients. Arch Surg 146, 794-801 (2011).

7. Institute of Public Health of Serbia: Health Statistical Yearbook of Republic of Serbia, 2015. Available at http://www.batut.org.rs/download/publikacije/pub2015.pdf

8. Mansur, A., Klee, Y., Popov, A. F., Erlenwein, J., Ghadimi, M., Beissbarth, T., Bauer, M., Hinz, J.: Primary bacteraemia is associated with a higher mortality risk compared with pulmonary and intra-abdominal infections in patients with sepsis: A prospective observational cohort study. BMJ Open 5, e006616 (2015).

9. Liesenfeld, O., Lehman, L., Hunfeld, K. P., Kost, G.: Molecular diagnosis of sepsis: New aspects and recent developments. Eur J Microbiol Immunol (Bp) 4, 1-25 (2014).

10. Gaynes, R., Richards, C., Edwards, J., Emori, T. G., Horan, T., Alonso-Echanove, J., Fridkin, S., Lawton, R., Peavy, G., Tolson, J.: Feeding back surveillance data to prevent hospital-acquired infections. Emerg Infect Dis 7, 295-298 (2001).

11. World Health Organization: WHO Guidelines on Hand Hygiene in Health Care. WHO, Geneva, 2009. Available at http://www.who.int/gpsc/5may/tools/9789241597906/en

12. American Society of Anesthesiologists Task Force on Central Venous Access, Rupp, S. M., Apfelbaum, J. L., Blitt, C., Caplan, R. A., Connis, R. T., Domino, K. B., Fleisher, L. A., Grant, S., Mark, J. B., Morray, J. P., Nickinovich, D. G., Tung, A.: Practice guidelines for central venous access. Anesthesiology 116, 539-573 (2012).

13. Horan, T. C., Andrus, M., Dudeck, M. A.: CDC/NHSN surveillance definition of health care-associated infection and criteria for specific types of infections in the acute care setting. Am J Infect Control 36, 309-32 (2008).

14. Hansen, S., Sohr, D., Geffers, C., Astagneau, P., Blacky, A., Koller, W., Morales, I., Moro, M. L., Palomar, M., Szilagyi, E., Suetens, C., Gastmeier, P.: Concordance between European and US case definitions of healthcare-associated infections. Antimicrob Resist Infect Control 1, 28 (2012). 
15. Djuric, O., Markovic-Denic, L., Jovanovic, B., Bumbasirevic, V.: Agreement between CDC/NHSN surveillance definitions and ECDC criteria in diagnosis of healthcareassociated infections in Serbian trauma patients. PLoS One 13, e0204893 (2018).

16. Horan, T. C., Emori, T. G.: Definitions of key terms used in the NNIS System. Am J Infect Control 25, 112-116 (1997).

17. Justiniano, C. F., Evans, D. C., Cook, C. H., Eiferman, D. S., Gerlach, A. T., Beery, P. R., Lindsey, D. E., Saum, G. E., Murphy, C. V., Miller, S. F., Papadimos, T. J., Steinberg, S. M., Stawicki, S. P.: Comorbidity-polypharmacy score: A novel adjunct in postemergency department trauma triage. J Surg Res 181, 16-19 (2013).

18. Clinical and Laboratory Standards Institute: Performance Standards for Antimicrobial Susceptibility Testing: 24th Informational Supplement, M100-S24. CLSI, Wayne, PA, 2014.

19. DeLong, E. R., DeLong, D. M., Clarke-Pearson, D. L.: Comparing the areas under two or more correlated receiver operating characteristic curves: A nonparametric approach. Biometrics 44, 837-845 (1988).

20. El-Masri, M. M., Hammad, T. A., McLeskey, S. W., Joshi, M., Korniewicz, D. M.: Predictors of nosocomial bloodstream infections among critically ill adult trauma patients. Infect Control Hosp Epidemiol 25, 656-663 (2004).

21. Mathur, P., Varghese, P., Tak, V., Gunjiyal, J., Lalwani, S., Kumar, S., Misra, M. C.: Epidemiology of blood stream infections at a level-1 trauma care center of India. J Lab Physicians 6, 22-27 (2014).

22. Mitharwal, S. M., Yaddanapudi, S., Bhardwaj, N., Gautam, V., Biswal, M., Yaddanapudi, L.: Intensive care unit-acquired infections in a tertiary care hospital: An epidemiologic survey and influence on patient outcomes. Am J Infect Control 44, e113-e117 (2016).

23. Lazarus, H. M., Fox, J., Burke, J. P., Lloyd, J. F., Snow, G. L., Mehta, R. R., Evans, R. S., Abouzelof, R., Taylor, C., Stevens, M. H.: Trauma patient hospital-associated infections: Risks and outcomes. J Trauma 59, 188-194 (2005).

24. Lazarus, H. M., Fox, J., Lloyd, J. F., Evans, R. S., Abouzelof, R., Taylor, C., Pombo, D. J., Stevens, M. H., Mehta, R., Burke, J. P.: A six-year descriptive study of hospital-associated infection in trauma patients: Demographics, injury features, and infection patterns. Surg Infect (Larchmt) 8, 463-473 (2007).

25. Giamberardino, H. I., Cesário, E. P., Carmes, E. R., Mulinari, R. A.: Risk factors for nosocomial infection in trauma patients. Braz J Infect Dis 11, 285-289 (2007).

26. Papia, G., McLellan, B. A., El-Helou, P., Louie, M., Rachlis, A., Szalai, J. P., Simor, A. E.: Infection in hospitalized trauma patients: Incidence, risk factors, and complications. J Trauma 47, 923-927 (1999).

27. Centers for Disease Control and Prevention: National Healthcare Safety Network Device Associated Module: Central Line Associated Bloodstream Infection Event, 2010. Available at http://www.cdc.gov/nhsn/PDFs/pscManual/4PSC_CLABScurrent.pdf

28. LeMaster, C. H., Schuur, J. D., Pandya, D., Pallin, D. J., Silvia, J., Yokoe, D., Agrawal, A., Hou, P. C.: Infection and natural history of emergency department-placed central venous catheters. Ann Emerg Med 56, 492-497 (2010).

29. Theodoro, D., Olsen, M. A., Warren, D. K., McMullen, K. M., Asaro, P., Henderson, A., Tozier, M., Fraser, V.: Emergency department central line-associated bloodstream infections (CLABSI) incidence in the era of prevention practices. Acad Emerg Med 22, 10481055 (2015). 
30. Baron, B. J., Nguyen, A., Stefanov, D., Shetty, A., Zehtabchi, S.: Clinical value of triage lactate in risk stratifying trauma patients using interval likelihood ratios. Am J Emerg Med 36, 784-788 (2018).

31. del Portal, D. A., Shofer, F., Mikkelsen, M. E., Dorsey, P. J., Gaieski, D. F., Goyal, M., Synnestvedt, M., Weiner, M. G., Pines, J. M.: Emergency department lactate is associated with mortality in older adults admitted with and without infections. Acad Emerg Med 17, 260-268 (2010).

32. Ljungström, L., Pernestig, A. K., Jacobsson, G., Andersson, R., Usener, B., Tilevik, D.: Diagnostic accuracy of procalcitonin, neutrophil-lymphocyte count ratio, C-reactive protein, and lactate in patients with suspected bacterial sepsis. PLoS One 12, e 0181704 (2017).

33. Lin, C. T., Lu, J. J., Chen, Y. C., Kok, V. C., Horng, J. T.: Diagnostic value of serum procalcitonin, lactate, and high-sensitivity $\mathrm{C}$-reactive protein for predicting bacteremia in adult patients in the emergency department. PeerJ 5, e4094 (2017).

34. Morales-Cartagena, A., Fernández-Ruiz, M., Lalueza, A., Lora-Tamayo, J., San Juan, R., López-Medrano, F., Origüen, J., Chaves, F., María Aguado, J.: Impact on mortality of adherence to evidence-based interventions in patients with catheter-related bloodstream infection due to methicillin-sensitive Staphylococcus aureus. Infect Dis (Lond) 16, 1-10 (2018).

35. Weiner, L. M., Webb, A. K., Limbago, B., Dudeck, M. A., Patel, J., Kallen, A. J., Edwards, J. R., Sievert, D. M.: Antimicrobial-resistant pathogens associated with healthcareassociated infections: Summary of data reported to the National Healthcare Safety Network at the centers for disease control and prevention, 2011-2014. Infect Control Hosp Epidemiol 37, 1288-1301 (2016).

36. Rosenthal, V. D., Maki, D. G., Mehta, Y., Leblebicioglu, H., Memish, Z. A., Al-Mousa, H. H., Balkhy, H., Hu, B., Alvarez-Moreno, C., Medeiros, E. A., Apisarnthanarak, A., Raka, L., Cuellar, L. E., Ahmed, A., Navoa-Ng, J. A., El-Kholy, A. A., Kanj, S. S., Bat-Erdene, I., Duszynska, W., Van Truong, N., Pazmino, L. N., See-Lum, L. C., Fernández-Hidalgo, R., Di-Silvestre, G., Zand, F., Hlinkova, S., Belskiy, V., Al-Rahma, H., Luque-Torres, M. T., Bayraktar, N., Mitrev, Z., Gurskis, V., Fisher, D., Abu-Khader, I. B., Berechid, K., Rodríguez-Sánchez, A., Horhat, F. G., Requejo-Pino, O., Hadjieva, N., Ben-Jaballah, N., García-Mayorca, E., Kushner-Dávalos, L., Pasic, S., Pedrozo-Ortiz, L. E., Apostolopoulou, E., Mejía, N., Gamar-Elanbya, M. O., Jayatilleke, K., de Lourdes-Dueñas, M., Aguirre-Avalos, G., International Nosocomial Infection Control Consortium: International Nosocomial Infection Control Consortium (INICC) report, data summary of 43 countries for 2007-2012. Device-associated module. Am J Infect Control 42, 942-956 (2014).

37. WHO Regional Office for Europe: Central Asian and Eastern European Surveillance of Antimicrobial Resistance. Annual report 2017. Denmark, 2018. Available at http:// www.euro.who.int/_data/assets/pdf_file/0005/354434/WHO_CAESAR_AnnualReport_ 2017.pdf?ua $=1$ 